\title{
Electrochemical Oxidation and Detection of Sodium Urate in Alkaline Media on a Copper Oxides Electrode
}

\author{
${ }^{1 *}$ MANEA, F; ${ }^{2}$ RADOVAN, C; ${ }^{3}$ SCHOONMAN, J \\ 1 "Politehnica" University of Timisoara, P-ta Victoriei, no.2, 300006, Timisoara, Romania, e-mail: \\ florica.manea@rectorat.utt.ro \\ ${ }^{2}$ West University of Timisoara, Str.Pestalozzi, no.16, 300115, Romania \\ ${ }^{3}$ Delft University of Technology, 2600 GA Delft, The Netherlands
}

\begin{abstract}
Electrochemical behaviour of copper oxides electrode in the presence of sodium urate was investigated. The correlation between the anodic oxidation and the amperometric detection of sodium urate in the alkaline medium on copper oxides electrode was analysed by cyclic voltammetry $(\mathrm{CV})$ and electrochemical impedance spectroscopy (EIS) measurements. The intereference from sodium tartrate presence in the aqueous alkaline solution was tested. Copper oxides electrodes can be used successfully for amperometric detection of both sodium urate and mixture of urate and tartrate as a cumulative response, in alkaline media, the target compound did not interfere each other. @JASEM
\end{abstract}

The monitoring of urate is required in a variety of investigations and environmental applications (Abuja, 1999; Basu et al., 2005; Hegazi, 2001; Lipkowitz et al., 2001; Proctor, 1970; Tebbut, 1983 ). Urate is produced as a result of purine degradation and high serum levels of uric acid are associated with an increased risk of developing gout, hypertension, cardiovascular disease and renal failure [Basu et al., 2005]. Related to the environmental applications, although, at the present there are no legal limits for urate in wastewaters, this organic can be regarded as representative class included in organic load parameter from municipal wastewater especially, expressed as COD index [Tebbut, 1983] that requires monitoring for wastewater quality control. There are electrochemical methods available for analytical determination of urate based on amperometric detection, i.e., using an uricase enzyme electrode and enzyme-free electrode (Janchen et al., 1983). A survey of the literature shows that several studies (Härtinger and Doblhofer, 1995; Jović and Jović, 2003; Lorimer et al., 2004) have been devoted to the investigation of the electrochemical behaviour of copper electrode in alkaline media. Copper oxides electrodes have been widely employed in the detection and the determination of a large number of

\section{MATERIALS AND METHODS}

Electrochemical measurements were performed in a Metrohm glass cell equipped with a three electrode system. This electrode system consists of a stationary horizontal copper disk electrode (working electrode) embedded in an insulating Teflon rod, which was fixed in a holder, a platinum plate as counter electrode, and a SCE (saturated calomel electrode) as

*-Corresponding author. Tel.:+40-256-403070; fax:+40-256-220369

e-mail address: florica.manea@,rectorat.utt.ro organic compounds (e.g., carbohydrates, aminoacids, sulfur-organics), with good sensitivity and reproducibility (Chen and Hibbert, 1997; Lee et al., 1999; Luo et al., 1990; Luo and Baldwin, 1995; Mho and Johnson, 2001Stulik and Pacakova, 1988; Torto et al., 1999). The copper oxides electrode can acts as a catalyst for the oxidation of electroactive organics by $\mathrm{Cu}$ (III) species (Marioli and Kuwana, 1992). This species has generally been considered to be uncommon oxidation state. In spite of this, in particular cases, $\mathrm{Cu}$ (III) occurs in the anodic potential range prior to $\mathrm{O}_{2}$ evolution and can be stabilised with formation of an electroactive species, envisaging a suitable approach for the amperometric detection (Nagy et al., 2001, Radovan and Manea, 2002). This paper examines the possibility of the oxidation of urate in order to evaluate it by amperometric detection. Supplementary experiments (cyclic voltammetry, chronoamperometry, electrochemical impedance spectroscopy) revealed the activation process of copper oxides electrode in the anodic potential range suitable for electrochemical detection of urate (0.4-0.6 V vs $\mathrm{SCE})$. Also, the possible interference of organics (e.g. tartrate) on the amperometric signal was tested.

reference electrode, respectively. The copper disk (advanced purity material) with the diameter of 2.8 $\mathrm{mm}$ was used. All chemicals were of analytical grade purchased from Merck. A stock $1 \mathrm{~mol} \cdot \mathrm{l}^{-1}$ sodium hydroxide solution was prepared with double distilled water. The sample $0.1 \mathrm{~mol} \cdot \mathrm{l}^{-1} \mathrm{NaOH}$ solutions were 
prepared at the same and were also used as supporting electrolyte for all measurements. The used solutions were non-dearated and the working temperature was $22 \pm 0.5{ }^{\circ} \mathrm{C}$. In order to obtain a freshly cleaned copper electrode surface, the copper electrode was polished in two steps with alumina powder $(0.25$ and $0.1 \mu \mathrm{m}$ dimensions $)$ suspended in distilled water, and carefully washed with double distilled water. The copper oxides-copper electrode was preformed in $0.1 \mathrm{M} \mathrm{NaOH}$ solution, by cyclic repeated scans, e.g. five repeated scans in a potential range of $-0.221 \mathrm{~V}$ to $+1 \mathrm{~V}$ (first switch potential) and $-1 \mathrm{~V}$ (second switch potential) vs. SCE and used further for the detection experiments. The oxidation of $2 \mathrm{mM}$ urate on copper oxides electrode was achieved in two variants, i.e., by repetitive cyclic voltammetry ( 5 scans) in the fore mentioned potential range, and by preanodization of copper electrode at controlled potential of $+2.5 \mathrm{~V}$ vs SCE for the time of 2 minutes in $0.1 \mathrm{M} \mathrm{NaOH}$ and $2 \mathrm{mM}$ urate solution.

The voltammograms and chronoamperograms were obtained using an Autolab PGstat 20 EcoChemie system controlled by a PC running 4.8 version GPES Software. For complementary correlation, the oxidation of urate on copper oxides-copper electrode was investigated by EIS experiments, which were made with an FRA module-containing Autolab
PGstat EcoChemie system controlled by a PC. The impedance measurements were carried out over a frequency range of $0.1 \mathrm{~Hz}$ to $10^{3} \mathrm{~Hz}$. The measurements were made continuously at each potential value correlated with the cyclic voltammogram. Fitting of the Impedance Spectra was done using the Zview program.

\section{RESULTS AND DISCUSSION}

The studies related to the oxidation of urate are achieved using cyclic voltammetry. The direct oxidation of urate on copper oxides electrode was achieved by the repetitive cyclic voltammetry in 0.1 $\mathrm{M} \mathrm{NaOH}$ and $2 \mathrm{mM}$ urate solution in the potential range from -1.00 to $+0.75 \mathrm{~V}$ vs SCE. The potential range for direct oxidation of urate is $0.2-0.6 \mathrm{~V}$ vs SCE, which is the same as carbohydrates (Radovan and Manea, 2002). Figure 1 shows cyclic voltammograms obtained by successive scans in the delimited potential range from 0.00 to $+0.75 \mathrm{~V}$ vs SCE for copper oxides electrode after its using under the same conditions but in the extended potential range prior mentioned. The anodic wave at $+0.6 \mathrm{~V}$ vs SCE attributed to the direct oxidation of $2 \mathrm{mM}$ urate with the involvement of $\mathrm{Cu}$ (III) species decreases with the number of scans. After the second scan, the curves are stabilised.

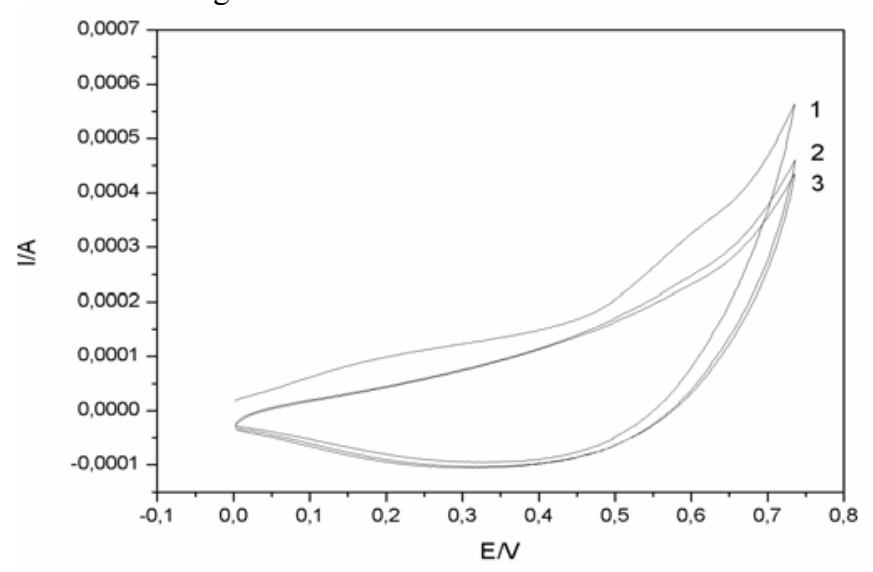

Fig 1. Cyclic voltammograms of copper oxides-copper electrode in $0.1 \mathrm{M} \mathrm{NaOH}$ and 2 $\mathrm{mM}$ urate after copper electrode cycling ( 5 scans) under the same conditions but in the extended potential range $(-0.221 \rightarrow+0.75 \rightarrow-1.00 \mathrm{~V}$ vs SCE); starting potential: $0.00 \mathrm{~V}$ vs SCE; potential range: $0.00 \rightarrow+0.75 \rightarrow 0.00 \mathrm{~V}$ vs SCE; scan rate: $0.05 \mathrm{~V} / \mathrm{s} ; 1-1^{\text {st }}$ scan, $2-3^{\text {rd }}$ scan, $3-5^{\text {th }}$ scan

The indirect oxidation of $2 \mathrm{mM}$ urate involving oxygen evolution was achieved by preanodization at $+2.5 \mathrm{~V}$ vs SCE for the time of 2 minutes using chronoamperometry technique (the signal was not recorded). Under these conditions, the major process of the overall oxidation process is oxygen evolution that leads to copper oxides layers with loose surface structure [Nagy et al., 2001] and the indirect oxidation of urate. The cyclic voltammograms obtained in the delimited potential range after the indirect oxidation are shown in Figure 2. At the first scan, the anodic wave at $+0.6 \mathrm{~V}$ vs SCE is not observed, this aspect could be explain by the prior major process of oxygen evolution that occurred by preanodization. After the first scan, the anodic wave appeared with the increase of the corresponding 
cathodic peak. This aspect could be attributed to the quasi-reversible redox process that occurs in this anodic potential range. The anodic wave could be due to the generation of the possible active form of $\mathrm{Cu}$ (III) species and anodic oxidation of the urate followed and sustained by a quasi-reversible reduction both of oxidation product and $\mathrm{Cu}$ (III) species, manifested in the nearly same positive potential range on the backward branch of the voltammograms.

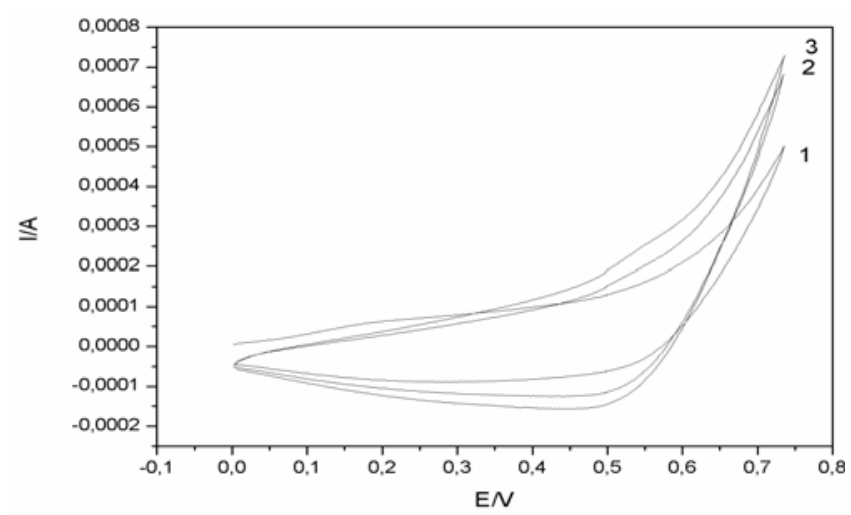

Fig 2. Cyclic voltammograms of copper oxides-copper electrode in $0.1 \mathrm{M}$ $\mathrm{NaOH}$ and $2 \mathrm{mM}$ urate after preanodization of copper electrode at $+2.5 \mathrm{~V}$ vs SCE for the time of 2 minutes; starting potential: $0.00 \mathrm{~V}$ vs SCE; potential range: $0.00 \rightarrow+0.75 \rightarrow 0.00 \mathrm{~V}$ vs SCE; scan rate: $0.05 \mathrm{~V} / \mathrm{s} ; 1-1^{\text {st }}$ scan, $2-3^{\text {rd }}$ scan, $3-5^{\text {th }}$ scan

Figure 3 presents cyclic voltammograms obtained with the copper oxides electrode in $0.1 \mathrm{M} \mathrm{NaOH}$ solution at increasing concentrations of urate. The signal obtained for each concentration of urate was stable and reproducible. This characteristic was observed after repetitive cycles (5 cycles) with the same electrode. Signals were stable after the second cycle. The sensitivity of this sensor was studied by comparing the responses to different concentrations of urate. The linear relationship between current magnitude and urate concentration was observed, as it can be seen in the inset of Fig. 3. The sensitivity of the copper oxides electrode to the urate is about $0.0325 \mathrm{~A} \cdot \mathrm{mol}^{-1}$.

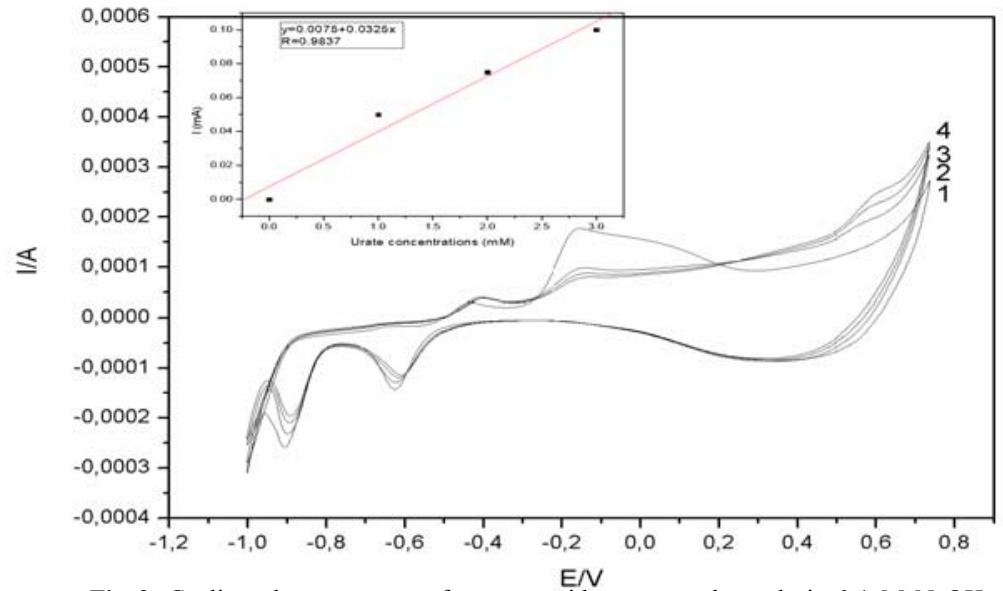

Fig 3. Cyclic voltammograms of copper oxides-copper electrode in $0.1 \mathrm{M} \mathrm{NaOH}$ (supporting electrolyte) and in the presence of various concentrations of urate: 1-0; 2-1mM; 3-2 $\mathrm{mM}$; 4-3 $\mathrm{mM}$; starting potential:-0.221V vs SCE; potential range: $0.221 \rightarrow+0.75 \rightarrow-1 \mathrm{~V}$ vs SCE; scan rate: $0.05 \mathrm{~V} / \mathrm{s} ; 5^{\text {th }}$ scan 
A supplementary test related to the interferences from other electroactive substances, which could co-exist for example as organic load in wastewaters represented by COD index confirms the quality of the copper oxides-copper sensor in alkaline media for urate detection in the presence of tratrate. This compounds is only one of many other interference candidates but it is relevant due to its oxidation potential is the same to that of urate. In Fig. 4 are compared two cyclic voltammograms i.e., in the presence of $2 \mathrm{mM}$ urate (curve 1) and in the mixture of both compounds at the same concentration (curve 2 ). In the detection region from +0.4 to +0.6 vs $\mathrm{SCE}$ the amperometric signals were higher and corresponded to cumulative quantitative characteristics of the both target compounds. These aspects suggest the possibilities for an overall quantitative evaluation of so-called organic load expressed as COD index from polluted water samples.

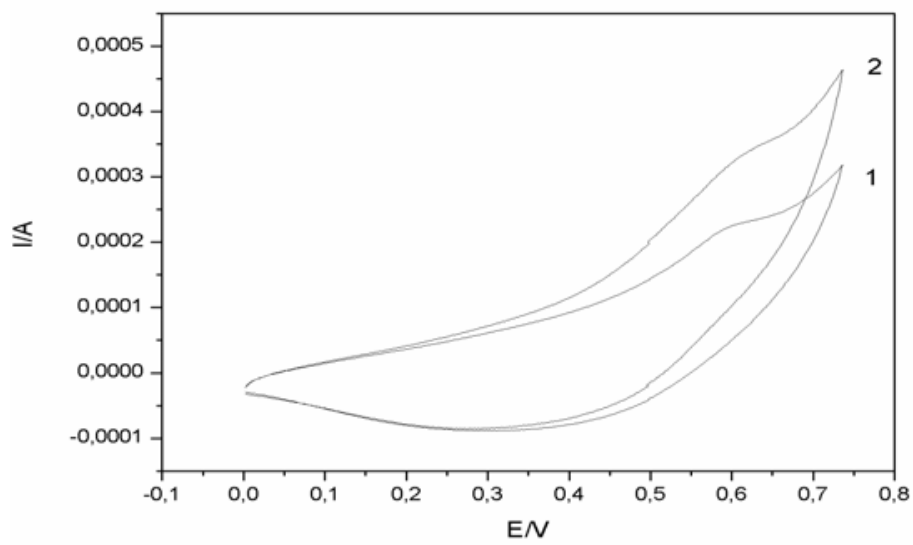

Fig 4. Cyclic voltammograms of copper oxides electrode in $0.1 \mathrm{M} \mathrm{NaOH}$ as supporting electrolyte and in the presence of : $2 \mathrm{mM}$ urate (curve 1);2mM urate and $2 \mathrm{mM}$ tartrate (curve 2); starting potential: $0.00 \mathrm{~V}$ vs SCE; potential range: $0.00 \rightarrow+0.75 \rightarrow 0 \mathrm{~V}$ vs $\mathrm{SCE}$; scan rate: $0.05 \mathrm{~V} / \mathrm{s} ; 5^{\text {th }} \mathrm{scan}$

In order to study the effect of urate on the electrochemical behaviour of copper electrodes in aqueous alkaline solution, impedance measurements at different potential values were performed. Impedance measurements of a copper electrode were performed with bias steps of $100 \mathrm{mV}$ starting at the potential value of $+0.4 \mathrm{~V}$ vs SCE going towards more positive potential $(+0.6 \mathrm{~V}$ vs SCE $)$ which precedes $\mathrm{O}_{2}$ evolution, in the frequency range from $10^{3} \mathrm{~Hz}$ to $0.1 \mathrm{~Hz}$. Before the impedance measurements, the copper electrode was running by $\mathrm{CV}$ in the absence and presence of $2 \mathrm{mM}$ urate. Figure 5 shows the Nyquist plots of a copper electrode in $0.1 \mathrm{M} \mathrm{NaOH}$ with and without urate addition. These EIS spectra were fitted to the equivalent circuit shown in Figure 6.

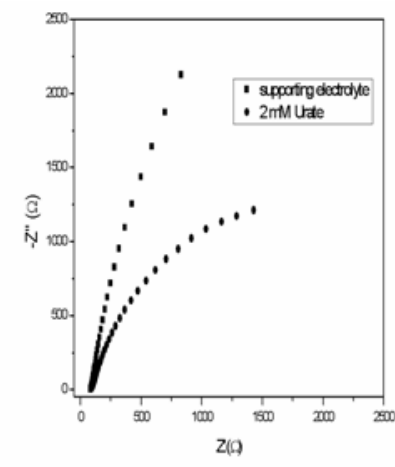

a)

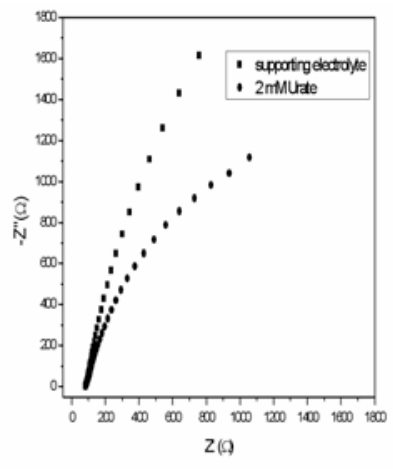

b)

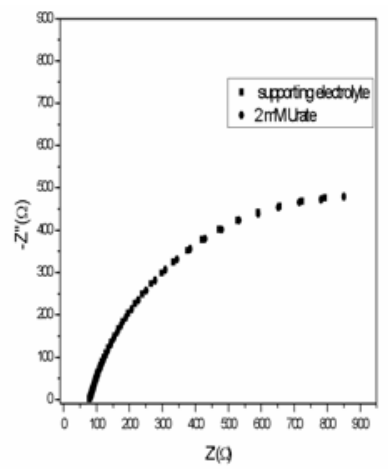

c)

Fig 5. The experimental Nyquist diagrams at different potential value of a copper electrode in $0.1 \mathrm{M} \mathrm{NaOH}$

*_Corresponding author. $\begin{gathered}\text { in absence of } \\ \text { * }\end{gathered}$

e-mail address: florica.manea@,rectorat.utt.ro 
These spectra can be related to the overall anodic and cathodic redox electrochemical processes. It is attributed to a charge-transfer resistance (R2) in parallel with a double layer capacitance C1. R2 reflects the dissolution rate of copper oxides in alkaline solution and R1 is the ohmic resistance of electrolyte. The smaller R2, the faster the dissolution reaction. The interfacial process is clearly limited by diffusion phenomena by presence of the "infinite"
Warburg element. The impedance of this element is expressed as:

$$
Z_{W}=\frac{1}{Y_{0}} \sqrt{(j \omega)} \quad \text { (1), where } \mathrm{Y}_{0} \text { is }
$$

Warburg coefficient that depends on the diffusion coefficient, surface area of the electrode, number of the electrons transferred and the bulk concentrations of the diffusing species.

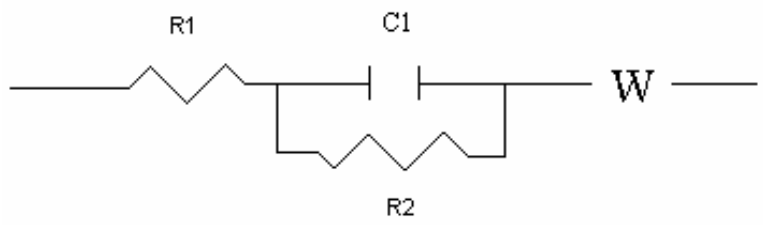

Fig 6. The equivalent circuits to fit the EIS for copper oxide electrodes in the absence/presence of urate in aqueous $0.1 \mathrm{M} \mathrm{NaOH}$ solution

Naturally, the charge transfer resistance decreased with the potential value increase, at the potential value of 0.6 vs SCE having almost the same value both in the presence and absence of urate. The presence of urate decreased the charge-transfer resistance value (table 1), indicates that the kinetics of charge transfer is favoured. This observation is in agreement with current dependence on potential in this range of the cyclic voltammogram (Figure 3). The values of the parameters obtained from the fits are gathered in Table 1. Taking into account the evolution of Warburg element related to the potential value and the presence of urate, it could be noticed that diffusion process occurred both in the presence and the absence of urate. Thus, these findings could prove the possibility of $\mathrm{Cu}$ (III) presence suggested by some authors (Marioli and Kuwana, 1992; Nagy et al., 2001, Radovan and Manea, 2002) and especially the involving of this species in the mediated oxidation of urate. The increasing of the potential value leads to the higher double layer capacitance and the smaller value of the Warburg coefficient, which could be due to decreasing of the bulk diffusing species concentration. Also, the existence of urate as bulk diffusing species is proved as higher value of the Warburg coefficient in the presence of urate versus in its absence.

Table 1. Values of EC elements (Fig. 4) in $0.1 \mathrm{M} \mathrm{NaOH}$ solution

\begin{tabular}{cccccc}
\hline Presence of urate & $\begin{array}{c}\mathrm{E} \\
(\mathrm{V}) / \mathrm{SCE}\end{array}$ & $\begin{array}{c}\mathrm{R} 1 \\
(\Omega)\end{array}$ & $\begin{array}{c}\mathrm{R} 2 / 10^{3} \\
\Omega\end{array}$ & $\mathrm{C} 2 / 10^{-6} \mathrm{~F}$ & $\begin{array}{c}\mathrm{Y}_{0} / 10^{2} \\
\Omega \mathrm{s}^{0.5}\end{array}$ \\
\hline no & 0.4 & 77.83 & 9.67 & 6.9 & 2.36 \\
yes & 0.4 & 85.22 & 2.40 & 7.0 & 3.72 \\
no & 0.5 & 75.73 & 6.93 & 9.3 & 2.60 \\
yes & 0.5 & 79.60 & 2.60 & 9.6 & 3.25 \\
no & 0.6 & 73.20 & 0.88 & 13.3 & 1.63 \\
yes & 0.6 & 75.40 & 0.90 & 12.6 & 2.07 \\
\hline
\end{tabular}

As conclusions, in this study it was evidenced the potential range of direct oxidation of urate with the involvement possibility of $\mathrm{Cu}$ (III) species in alkaline media by direct correlation of the shape of the 
voltammograms obtained in delimited anodic potential range with the EIS results. This aspect confirmed by the evolution of the charge-transfer resistance in the detection potential range. The urate detection was not hindered by the tartrate presence while the detection potential values were the same, a cumulative response being obtained. The advantage of this detector type is evident and it appears as useful electrochemical inexpensive and easily renewable sensor, the envisaged aim being its using as amperometric detector for either oxidable organics from polluted waters or urate using enzyme-free electrode.

\section{REFERENCES}

Abuja, P M (1999). Ascorbate prevents prooxidant effects of urate in oxidation of human low density lipoprotein. FEBS Lett 446:305-308

Basu, J; Mikhail, M S; Ahn, C W; Furguiele, J; Ho, Y G; Burk, R D; Palan, R P; Romney, S L (2005). Plasma uric acid levels in woman with cervical intraepithelial neoplasia. Nutrition and Cancer 51: 2531

Chen, Z L; Hibbert, D B (1997). Simultaneous amperometric and potentiometric detection of sugars, polyols and carboxylic acids in flow systems using copper wire electrodes. J Chromatogr A 766: 27-38

Hegazi, A N (2001). A new concept for urate in human serum: Enzymatic assay of total urate (protein bound \&loosely associated) in serum using 3,5dichloro-2-hydroxybenzenesulphonic acid/4aminophena zone chromogenic system. Arthritis Res 3: E003

Härtinger, S; Doblhofer, K (1995). The electrochemical interface between copper (111) and aqueous electrolytes. J Electroanal Chem 380: 185192

Janchen, M; Walzel, G; Neef, B; Scheller, F; Kuhn, M; Pfeiffer, D; Sojka, W; Jaross, W (1983). Uric acid determination in dilute serum with an enzyme electrochemical and enzyme-free sensor. Bimed Biochim Acta 42: 1055-10065

Jović, V D; Jović, B M (2003). EIS and differential capacitance measurements onto single crystal faces in different solutions Part II: $\mathrm{Cu}$ (111) and $\mathrm{Cu}(100)$ in 0.1 M NaOH. J Electroanal Chem 541: 13-19

Lee, K H; Ishikawa, T; McNiven, S J; Nomura, Y; Hiratsuka, A; Sasaki, S; Arikawa, Y; Karube, I (1999). Evaluation of chemical oxygen demand (COD) based on coulometric determination of electrochemical oxygen demand (EOD) using a surface oxidized copper electrode. Anal Chim Acta 398: 161-167

Lipkowitz, S M; Leal-Pinto, E; Rappoport, J Z; Najfeld, V; Abramson, G R (2001). Functional reconstitution, membrane targeting, genomic structure, and chromosomal localization of a human urate transporter. J Clin Invest 107:1103-1114

Lorimer, J P; Mason, T J; Plattes, M ; Walton, D J (2004). Passivation phenomena during sonovoltammetric studies on copper in strongly alkaline solutions. J Electroanal Chem 568: 379-385

Luo, P; Prabhu, S V; Baldwin, R P (1990). Constant Potential Amperometric Detection at a Copper-Based Electrode: Electrode Formation and Operation. Anal Chem 62: 752-760

Luo, M Z; Baldwin, R P (1995). Characterization of carbohydrate oxidation at copper electrodes. J Electroanal Chem 387: 87-96

Marioli, J M; Kuwana, T (1992). Electrochemical characterization of carbohydrate oxidation at copper electrodes. Electrochim Acta 37: 1187-1193

Mho, S; Johnson, D C (2001). Electrocatalytic response of carbohydrates at copper-alloy electrodes. J Electroanal Chem 500: 524-532

Nagy, L; Nagy, G; Hajos, P (2001). Copper electrode based amperometric detector cell for sugar and organic acid measurements. Sens and Actuators B 76: 494-500

Proctor, P (1970). Similar functions of uric acid and ascorbate in man. Nature 228: 868-872

Radovan, C; Manea, F (2002). Some aspects concerning the electrochemical detection of a simulated organic load. Chem Bull "Politehnica" Univ 47: 21-26

Stulik, K; Pacakova, V (1988). Amperometric flow detection with a copper working electrode-response mechanism and application to various compounds. Talanta 35: 455-461

Tebbut, T H Y (1983). Principles of water quality control. Pergamon Press, Oxford

Torto, N; Ruzgas, T; Gorton, L (1999). Electrochemical oxidation of mono- and disaccharides at fresh as well as oxidized copper electrodes in alkaline media. J Electroanal Chem 464: 252-260 